ARTICLE

Received 30 Jun 2014 | Accepted 15 Dec 2014 | Published 19 Jan $2015 \quad$ DOl: 10.1038/ncomms7105

\title{
Prevalent and distinct spliceosomal 3'-end processing mechanisms for fungal telomerase RNA
}

\author{
Xiaodong $\mathrm{Qi}^{1, \star}$, Dustin P. Rand ${ }^{1, \star}$, Joshua D. Podlevsky ${ }^{1}$, Yang $\mathrm{Li}^{1, \dagger}$, Axel Mosig ${ }^{2}$, Peter F. Stadler ${ }^{3}$ \\ \& Julian J.-L. Chen ${ }^{1}$
}

Telomerase RNA (TER) is an essential component of the telomerase ribonucleoprotein complex. The mechanism for TER $3^{\prime}$-end processing is highly divergent among different organisms. Here we report a unique spliceosome-mediated TER $3^{\prime}$-end cleavage mechanism in Neurospora crassa that is distinct from that found specifically in the fission yeast Schizosaccharomyces pombe. While the S. pombe TER intron contains the canonical $5^{\prime}$-splice site GUAUGU, the N. crassa TER intron contains a non-canonical 5'-splice site AUAAGU that alone prevents the second step of splicing and promotes spliceosomal cleavage. The unique N. crassa TER 5'-splice site sequence is evolutionarily conserved in TERs from Pezizomycotina and early branching Taphrinomycotina species. This suggests that the widespread and basal N. crassa-type spliceosomal cleavage mechanism is more ancestral than the S. pombe-type. The discovery of a prevalent, yet distinct, spliceosomal cleavage mechanism throughout diverse fungal clades furthers our understanding of TER evolution and non-coding RNA processing.

\footnotetext{
${ }^{1}$ Department of Chemistry \& Biochemistry, Arizona State University, Tempe, Arizona 85287, USA. ${ }^{2}$ Department of Biophysics, Ruhr-University Bochum, D-44780 Bochum, Germany. ${ }^{3}$ Department of Computer Science, and Interdisciplinary Center for Bioinformatics, University of Leipzig, D-04107 Leipzig, Germany. ${ }^{\star}$ These authors contributed equally to this work. † Present address: Barrow Neurological Institute, Phoenix, Arizona 85013, USA. Correspondence and requests for materials should be addressed to J.J.-L.C. (email: jlchen@asu.edu).
} 
$\mathrm{T}$ he telomerase enzyme is responsible for the addition of telomeric DNA repeats onto the ends of eukaryotic chromosomes to maintain genome stability and cellular replicative capacity ${ }^{1}$. The core telomerase ribonucleoprotein (RNP) enzyme is composed of the catalytic telomerase reverse transcriptase (TERT) and telomerase RNA (TER) that contains a short region as the template for telomeric DNA repeat synthesis ${ }^{2}$. TERs are highly divergent across eukaryotic clades, differing immensely in nucleotide sequence, length and biogenesis pathway ${ }^{3,4}$. The diversity in TER biogenesis is evidenced by the different RNA polymerase (pol) machineries employed for transcription. While TER is transcribed by RNA pol II in most species, it is transcribed by RNA pol III in ciliates ${ }^{5-8}$. Furthermore, TERs from evolutionary separate groups of species associate with entirely unique groups of accessory proteins-the dyskerin complex and TCAB1 in vertebrates ${ }^{9,10}$; Est1, Est3 and Sm-ring in budding yeasts ${ }^{11-13}$; the dyskerin complex and POT1 in the plant Arabidopsis ${ }^{14,15}$ and $\mathrm{p} 50$ and p65 in ciliates ${ }^{16}$. These separate sets of TER accessory proteins are essential for localization, RNP assembly and TER $3^{\prime}$-end processing ${ }^{17}$.

The identification of the Schizosaccharomycetes pombe TER (SpoTER1) revealed a spliceosome-mediated cleavage mechanism for RNA $3^{\prime}$-end processing ${ }^{18-20}$. The nascent SpoTER1 transcript harbours a downstream intron followed by a second exon and a $3^{\prime}$-poly(A) tail. The mature SpoTER1 $3^{\prime}$-end is generated by spliceosomal cleavage at the $5^{\prime}$-splice site $\left(5^{\prime}\right.$-SS), removing the intron, second exon and $\operatorname{poly}(\mathrm{A})$ tail. This is in contrast to splicing-whereby the intervening intron sequence is excised and the neighbouring exons are joined ${ }^{21}$. The SpoTER1 intron itself uncouples these two tightly associated splicing steps by inhibiting the second transesterification step at the $3^{\prime}$-splice site ( $3^{\prime}$-SS). The second transesterification step is abated by a strong branch point sequence (BPS), an extended distance from the BPS to the $3^{\prime}$-SS and a weak polypyrimidine (Py) in the SpoTER1 intron ${ }^{19}$.

Here we report in $N$. crassa a spliceosome-mediated mechanism for TER $3^{\prime}$-end processing that is distinct from the S. prombe-type. The N. crassa-type TER spliceosome-mediated $3^{\prime}$-end cleavage relies exclusively on a unique $5^{\prime}$-SS AUAAGU, which alone is necessary and sufficient for inhibiting the second transesterification step in splicing. This is the first biologically functional instance of spliceosome-mediated cleavage relying exclusively on the $5^{\prime}$-SS. In addition, the $N$. crassa-type TER intron is pervasive across Ascomycota, found universally in TERs from filamentous fungi and select early branching fission yeasts. This preponderance and conservation among distinct, evolutionary distant and basal fungal species suggest that this mechanism is more ancestral than the $S$. pombe-type. The discovery of a unique spliceosome-mediated cleavage mechanism provides new insights into TER biogenesis and evolution.

\section{Results}

Neurospora crassa TER precursor harbours an intron. Neurospora crassa TER (NcrTER) was recently identified as a 2,049-nt RNA ${ }^{22}$. The $3^{\prime}$-end of the mature NcrTER was determined by rapid amplification of cDNA ends (RACE) from total RNA treated with poly(A) polymerase (Fig. 1a, lane 1). However, 3'-RACE with total RNA untreated with poly(A) polymerase generated two larger products (Fig. 1a, lane 2). While the smaller of these two products was not readily visible by ethidium bromide staining, it was discernable for gel extraction. Cloning and subsequent sequencing of the two PCR products revealed distinct NcrTER variants of $\sim 400$ and $470 \mathrm{nt}$ in length. The 470-nt product harboured a 74-nt intron sequence, while the 400-nt product lacked this intron with the two exons joined (Fig. 1b). This intervening sequence contained the conserved features of a canonical intron: $5^{\prime}$-SS, BPS and $3^{\prime}$-SS (Fig. 1c). The presence of an intron in NcrTER transcripts suggested that NcrTER undergoes spliceosome-mediated cleavage for $3^{\prime}$-end processing, similar to that discovered in the fission yeast SpoTER1 (ref. 18). Interestingly, the NcrTER intron $5^{\prime}$-SS AUAAGU is non-canonical, a stark contrast to the canonical $5^{\prime}-\overline{S S}$ GUAUGU sequence found in the SpoTER1 and mRNA introns ${ }^{18}$. We then determined the relative abundance of the three NcrTER variants by performing northern blot analysis of total RNA with probes targeting either the first or second exon. The 2,049-nt NcrTER was readily detected by northern blot, while the precursor and spliced variants were below the threshold for detection (Fig. 1d). This result suggests that the mature 2,049-nt NcrTER variant has far greater abundance, which is consistent with our initial identification of only the 2,049-nt NcrTER species in the purified telomerase holoenzyme ${ }^{22}$.

NcrTER-type intron is prevalent in Ascomycota fungal TERs. Analysis of 72 additional Pezizomycotina filamentous fungal TERs that we previously identified revealed the absolute conservation of the NcrTER-type intron in all species examined (Fig. 2a). All known Pezizomycotina TER introns contain the unique non-canonical $5^{\prime}$-SS AUAAGU sequence, the BPS sequence RCURAY that includes an invariant adenine residue as a putative branch point residue and the putative $3^{\prime}$-SS YAG (Supplementary Fig. 1). The $3^{\prime}$-SS was experimentally verified by 3'-RACE for N. crassa, Aspergillus nidulans and Mycosphaerella graminicola (Supplementary Fig. 1). The NcrTER 5'-SS AUAAGU is extremely conserved in filamentous fungal TERs and distinct from the canonical, yet less conserved $5^{\prime}$-SS GURNGU found in yeast TER introns from the fission yeast $S$. pombe as well as species from the budding yeast Candida and Hansenula genera ${ }^{18,23,24}$

We then investigated whether the $S$. pombe-type or the $N$. crassa-type TER intron is more ancestral. S. pombe belongs to the subphylum Taphrinomycotina that together with the other two subphyla Pezizomycotina and Saccharomycotina constitute the Ascomycota phylum. In addition to TER that we previously cloned from the basal Taphrinomycotina species Saitoella complicata $^{22}$, we identified two additional Taphrinomycotina TERs from Schizosaccharomyces octosporus and Schizosaccharomyces japonicus by a bioinformatics approach (see Methods). The $3^{\prime}$-ends and intron sequences of these newly identified TERs were experimentally determined by RACE. Surprisingly, there was a clear divide in the intron $5^{\prime}$-SS sequence among Taphrinomycotina TERs. The basal species $S$. complicate and $S$. japonicus harboured the $N$. crassa-type 5'-SS AUAAGU, while the later evolving species $S$. octosporus contained the S. pombe-type $5^{\prime}$-SS GURNGU (Fig. 2b). Thus, the more prevalent $N$. crassa-type intron appears to be more basal and thus ancestral than the $S$. pombe-type intron (Fig. 2c).

NcrTER-type $5^{\prime}$-SS promotes spliceosomal cleavage. The $5^{\prime}$-SS of the NcrTER and canonical mRNA introns differ with respect to their first and forth residues (Fig. 3a). Newman et al. ${ }^{25}$ demonstrated in yeast that an introduced G-to-A substitution at the first residue in an mRNA intron suppresses the second step, yet permits the first step of splicing. We thus hypothesized that the $5^{\prime}$-SS is sufficient for NcrTER $3^{\prime}$-end processing. To test this, we constructed a minimal exogenous expression cassette of the NcrTER gene. This minimal NcrTER expression cassette spanned a $3^{\prime}$-portion (residues 1,888-2,049) of exon 1, the intron (residues 2,050-2,123), exon 2 (residues 2,124-2,200) and the $500 \mathrm{bp}$ downstream flanking genomic sequence that contained a polyadenylation signal (Fig. 3b,c). This expression cassette also 
a

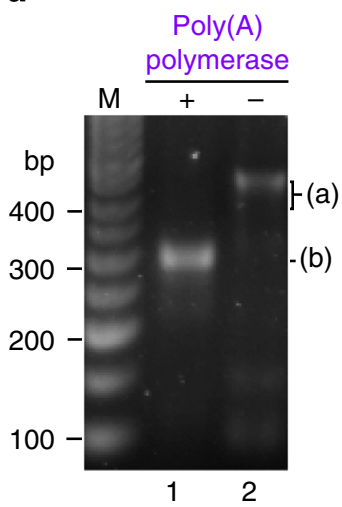

b

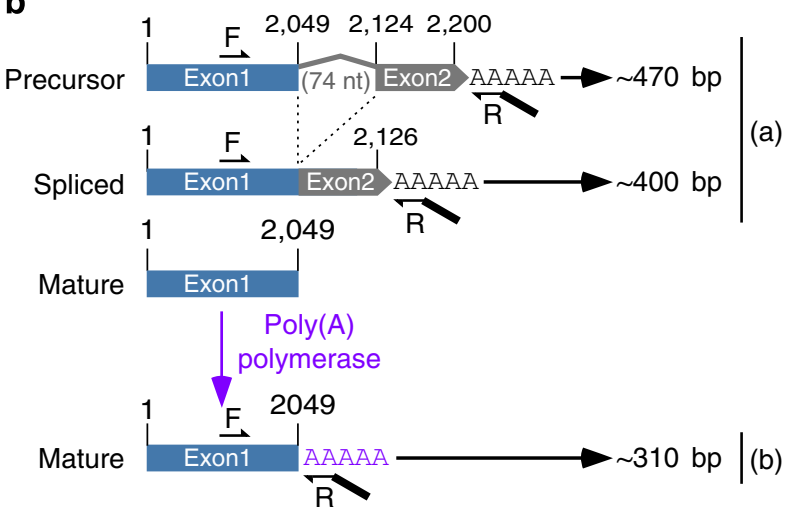

C

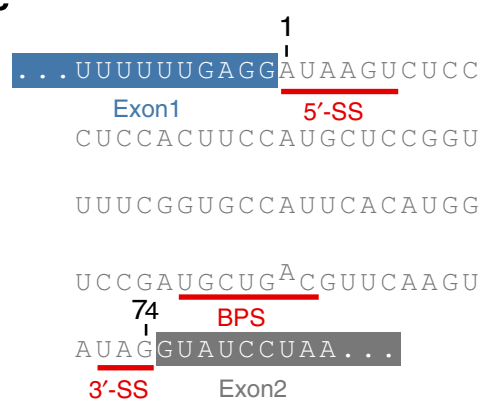

d

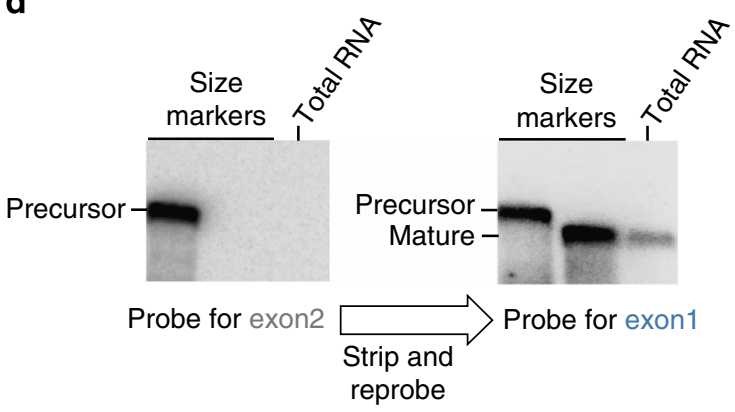

Figure 1 | Characterization of the NcrTER intron and $\mathbf{3}^{\prime}$-end processing variants. (a) $3^{\prime}$-RACE of NcrTER. Two distinct PCR products (a and b) were amplified from the RNA samples with $(+)$ or without $(-)$ poly $(A)$ polymerase treatment. A 50-bp ladder (M) was included with sizes denoted. (b) Schematic of NcrTER splicing variants. Exon 1 (blue box), 74-nt intron (grey line) and exon 2 (grey box) are denoted. Positions of forward (F) and reverse (R) primers used in RT-PCR are indicated. RT-PCR of the precursor and spliced RNA generated products (a) of 470 and 400 bp, respectively. RT-PCR of the matured RNA pretreated with poly(A) polymerase generated the 310 bp product (b). (c) Sequence of the NcrTER intron. The sequences of exon 1 (blue box) and exon 2 (grey box) flanking the NcrTER intron. The 5'-splice site (5'-SS), branch point sequence (BPS) and 3'-SS are denoted below the intron sequence. (d) Northern blot analysis of NcrTER splicing variants. Northern blot was probed for NcrTER exon 2 (grey) first and the stripped blot was re-probed for exon 1 (blue). In vitro transcribed precursor and mature NcrTER were loaded as size markers.

contained an eGFP gene as an internal control for RNA expression and normalization. NcrTER and eGFP mRNA were transcribed independently under separate $c c g$ - 1 (clock-controlled gene-1) promoters and were fused to the ccg-1 $5^{\prime}$-UTR sequence, generating ccg-1::NcrTER and ccg-1::eGFP, respectively. The $c c g-1$ promoter is a light induced, strong RNA pol II promoter commonly used for ectopic gene expression in the $N$. crassa model system ${ }^{26}$. Northern blot analysis was performed to determine the steady-state level of the NcrTER exon 1 fragment and eGFP mRNA with probes targeting the fused ccg- $15^{\prime}$-UTR sequence (Fig. 3b). The ccg-1::NcrTER construct generated a stable RNA product detectable by northern blot analysis (Fig. 3d, lane 3). This RNA product had the expected $3^{\prime}$-end corresponding to the mature NcrTER $3^{\prime}$-end, verified by $3^{\prime}$-RACE. Thus, the exogenous overexpressed minimal NcrTER $3^{\prime}$-fragment was processed by spliceosome-mediated cleavage, similar to the full-length endogenous NcrTER. RT-PCR was performed to detect the precursor and spliced RNA transcripts, which were below the detection limit of northern blot (Figs 1d and $3 \mathrm{~d}$ ). While the unspliced precursor and spliced NcrTER 3 -fragments were both detected, the precursor appeared to be much more abundant (Fig. 3e, lane 3). This is consistent with the RT-PCR results of the endogenous NcrTER precursor and spliced product (Fig. 1a, lane 2). Thus, our ccg-1::NcrTER expression cassette provides a minimal system for the functional analysis of spliceosome-mediated NcrTER $3^{\prime}$-end processing.

Mutations were introduced to the NcrTER intron in $5^{\prime}$-SS, BPS and $3^{\prime}$-SS to identify the element(s) necessary for NcrTER $3^{\prime}$-end processing (Fig. 3c). The $5^{\prime}$-SS_1 mutant with the first residue mutated from A to G reduced the level of mature NcrTER exon-1 RNA to $8 \%$ of wild type (Fig. 3d, lane 4 ), presumably by restoring the second step of splicing. Indeed, RT-PCR and subsequent sequencing of PCR products confirmed efficient splicing of the 5'-SS_1 mutant RNA (Fig. 3e, lane 4; Supplementary Fig. 2). In contrast, the $5^{\prime}$-SS_2 mutant with the fourth residue mutated from A to $\mathrm{U}$ only slightly reduced the level of mature NcrTER exon-1 RNA (Fig. 3d, lane 7) and did not significantly affect splicing efficiency (Fig. 3e, lane 7; Supplementary Fig. 2). Thus, the first adenosine residue in the NcrTER $5^{\prime}$-SS appears to be the key determinant for NcrTER $3^{\prime}$-end processing.

We then examined if the $5^{\prime}$-SS_1 mutant-which has complete splicing restored-could be converted for S. pombetype spliceosomal cleavage by extending the distance between the BPS and the $3^{\prime}$-SS (Supplementary Fig. 3). We generated a double mutant $5^{\prime}$-SS_la that, in addition to the $5^{\prime}$-SS_1 mutation, disrupted the $3^{\prime}$-SS (Fig. 3c). A triple mutant $5^{\prime}-\mathrm{SS} \_1 \mathrm{~b}$ further disrupted a downstream cryptic $3^{\prime}$-SS (Fig. 3c). Both $5^{\prime}$-SS_1a and $5^{\prime}$-SS_1b mutants effectively restored spliceosomal cleavage and accumulated mature RNA at levels comparable to the wild-type NcrTER intron (Fig. 3d, lanes 5 and 6), while reducing the splicing efficiency (Fig. 3e, lanes 5 and 6). RT-PCR and sequencing of the spliced RNA products from $5^{\prime}$-SS_1a and $5^{\prime}$-SS_1b mutants showed that, when the preferred $3^{\prime}$-SS was mutated, two different cryptic $3^{\prime}$-SSs were used instead with low efficiencies (Supplementary Fig. 2). Thus, disruption of the $N$. crassa-type spliceosomal cleavage in the $5^{\prime}-\mathrm{SS} \_1$ mutant was 


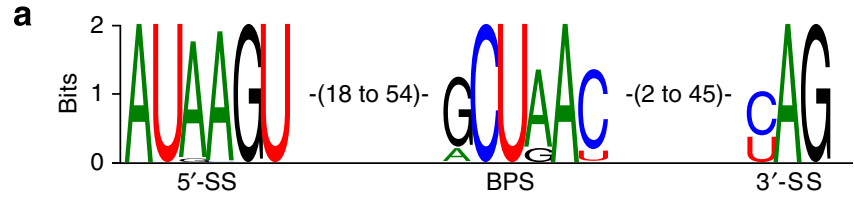

b

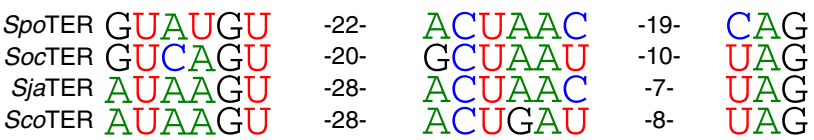

c

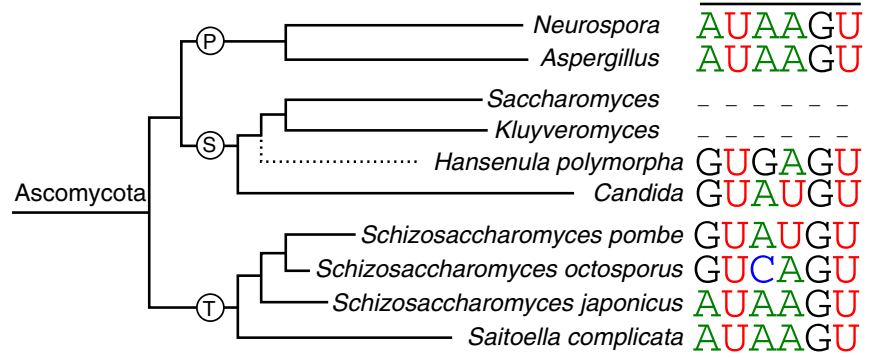

Figure 2 | Conservation and diversity of fungal TER intron sequence.

(a) Sequence conservation of filamentous fungal TER introns. Consensus sequences of $5^{\prime}$-SS, BPS and $3^{\prime}$-SS are derived from a multiple sequence alignment of 73 Pezizomycotina TER introns and presented using Sequence Logo. (b) Sequence conservation of four Taphrinomycotina TER introns. The aligned sequences of 5'-SS, BPS and 3'SS of TER introns from Schizosaccharomyces pombe (Spo), S. octosporus (Soc), S. japonicus (Sja) and Saitoella complicata (Sco) are shown. (c) Evolutionary relationship of fungal TER introns. The phylogenetic tree of select species from Pezizomycotina $(P)$, Saccharomycotina $(S)$ and Taphrinomycotina $(T)$ is based on a recent fungal six-gene phylogeny study (James et al. (2006); Liu et al. (2009)). Respective intron $5^{\prime}$-SS sequences from select species with known TER introns are shown. The length of the branch line does not reflect evolutionary distance. The evolutionary position of Hansenula polymorpha is tentative (dashed line).

rescued by extending the distance between the BPS and the $3^{\prime}$-SS to employ $S$. pombe-type spliceosomal cleavage.

To verify that the NcrTER $3^{\prime}$-end processing is indeed spliceosome-mediated and not through non-specific endo- or exo-nucleolytic RNA degradation, we generated two mutations, BPS_1 and No_intron. Mutant BPS_1 abolished splicing by mutating the essential adenosine residue of the BPS and generated exclusively precursor RNA, while mutant No_intron produced only the corresponding spliced RNA product by deleting the entire intron. As expected, BPS_1 and No_intron mutants failed to accumulate mature RNA, indicating that spliceosome-mediated processing is required to generate the mature $3^{\prime}$-end of NcrTER (Fig. 3d, lanes 8 and 9). RT-PCR confirmed the lack of splicing with the BPS_1 mutant and the expected RNA transcript from the No_intron mutant (Fig. 3e, lanes 8 and 9).

The NcrTER intron boundary $5^{\prime}$-AU/AG- $3^{\prime}$ is chimeric with a $3^{\prime}$-SS similar to the canonical GU/AG mRNA introns and a $5^{\prime}$-SS similar to rare AU/AC mRNA introns ${ }^{27-29}$. We investigated whether the NcrTER intron can be converted to an AU/AC intron for splicing. Mutations were introduced to convert the NcrTER intron 3'-SS UAG to UAC. Mutant 3'-SS_1 had the proximal 3'SS UAG changed to UAC, while mutant $3^{\prime}$-SS_1a had an additional mutation converting a downstream cryptic 3'-SS UAG to UAC (Fig. 3c). Mutant 3'-SS_1 showed a slightly decreased level of mature RNA at $80 \%$ of the wild type (Fig. 3f, lane 2) and effectively increased splicing efficiency (Fig. 3g, lane
2), confirmed by RT-PCR and sequencing of the spliced products (Supplementary Fig. 2). Mutant 3'-SS_1a further decreased spliceosomal cleavage to $60 \%$ of the wild type (Fig. 3f, lane 3) and a greater increase in splicing efficiency (Fig. 3g, lane 3). Thus, the NcrTER intron can be readily converted to an AU/AC splicing intron by mutating the proximal and distal $3^{\prime}$-SS UAG to UAC.

5'-SS AUAAGU converts an intron for spliceosomal cleavage. The NcrTER intron can be converted to favour splicing by a simple A-to-G substitution at the first adenine reside of the $5^{\prime}$-SS (Fig. 3). To further confirm that the NcrTER $5^{\prime}$-SS AUAAGU is the key determinate for spliceosomal cleavage, we performed a reciprocal experiment to convert an mRNA intron to the NcrTER-type intron for spliceosomal cleavage. We modified our ccg1::NcrTER expression cassette by replacing the NcrTER intron with an mRNA intron from the $c c g-1$ gene (Fig. $4 \mathrm{a}$ ).

Mutations were introduced to the ccg- 1 mRNA at $5^{\prime}$-SS, BPS and $3^{\prime}$-SS to identify the element(s) necessary for conversion to NcrTER-type spliceosomal cleavage (Fig. 4b). The replacement of the NcrTER intron in our minimal expression cassette with the ccg-1 mRNA intron resulted in severely reduced spliceosomal cleavage (Fig. 4c, lane 1) and markedly increased splicing efficiency (Fig. 4d, lane 1). In mutant $5^{\prime}$-SS_3, replacing the ccg-1 5'-SS with the NcrTER 5'-SS, by two G-to-A substitutions at the first and fourth positions of the $5^{\prime}$-SS, significantly increased the accumulation of mature NcrTER exon-1 fragment (Fig. 4c, lane 2) and reduced splicing efficiency (Fig. $4 \mathrm{~d}$, lane 2). Thus, the NcrTER 5'-SS AUAAGU alone appears to be sufficient to convert an mRNA intron for spliceosomal cleavage. Interestingly, sequencing the residual spliced products of the $5^{\prime}$-SS_3 mutant showed that a new $3^{\prime}$-SS CAC was utilized, similar to the AU/AC mRNA splicing introns (Supplementary Fig. 4a). In the presence of the $5^{\prime}$-SS AUAAGU, there is a shift from the $3^{\prime}$-SS YAG employed by GU/AG mRNA introns to YAC employed by AU/ AC mRNA introns. To minimize the 3'-SS YAC, we generated two double mutants, 5'-SS_3a and _3b, that changed the $3^{\prime}$-SS CAC employed by mutant $5^{\prime}$-SS_3 to CGC or CAU, respectively (Fig. 4b). As expected, preventing AU/AC mRNA splicing for each of these mutants resulted in far greater accumulation of mature NcrTER than the mutant 5'-SS_3 (Fig. 4c, lanes 3 and 4) and similarly reduced splicing efficiency (Fig. $4 \mathrm{~d}$, lanes 3 and 4). The residual spliced products from the mutants $5^{\prime}$-SS_3a and $5^{\prime}$-SS_3b arose from utilizing the $3^{\prime}$-SS CAG or UAG, respectively (Supplementary Fig. 4a). Thus, the $5^{\prime}-$ SS_3a and $5^{\prime}$-SS_3b mutants employed the same $3^{\prime}$-SS YAG as the wild-type NcrTER intron.

We next investigated whether a single substitution at the first residue of the ccg-1 5'-SS would convert the ccg-1 mRNA intron to the NcrTER intron. In contrast to mutant $5^{\prime}$-SS_3 that had both the first and fourth residues substituted and accumulated mature NcrTER, mutant 5'-SS_4 with only the first residue substituted failed to accumulate mature NcrTER above the level of the wild-type ccg-1 intron (Fig. 4c, lane 5). The double mutations $5^{\prime}$-SS_4a and $5^{\prime}$-SS_4b eliminated the $3^{\prime}$-SS CAC to prevent AU/AC splicing. The northern blot showed that mutant $5^{\prime}$-SS_4a did not increase mature RNA level (Fig. 4c, lane 6). However, mutant $5^{\prime}$-SS_4b had only a slight increase in mature NcrTER accumulation, which is relatively insignificant compared with the 5'-SS_3 mutant (Fig. 4c, lane 7). Thus, the ccg-1 mRNA intron requires a $5^{\prime}$-SS identical to the NcrTER-type intron for spliceosomal cleavage. The requirement of G-to-A substitutions for the first and fourth residues in the ccg-1 mRNA intron may relay on the first adenine substitution disrupting snRNA U1 interaction and the fourth position substitution compensating for this disruption. 
a

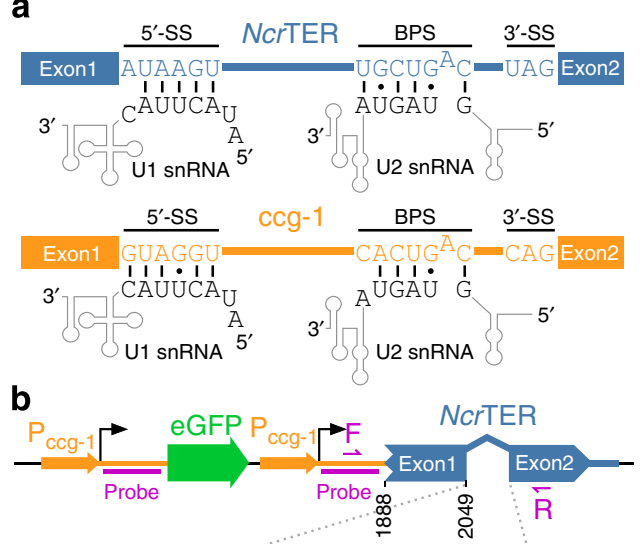

C

\begin{tabular}{|c|c|c|c|c|}
\hline & $5^{\prime}-S S$ & BPS & $\underline{3}$-SS & * \\
\hline NcrTER & AUAAGU & $\mathrm{UGCUG}^{\mathrm{A}} \mathrm{C}$ & $-\mathrm{UAG}-2$ & $\overline{U A G}$ \\
\hline 5'-SS_1 & $G \cdots \cdots$ & $\cdots \cdots$ & $\cdots$ & $\cdots$ \\
\hline $5^{\prime}-S S \_1 a$ & $G \cdots \cdots$ & $\cdots \cdots$ & $\cdot \mathrm{UC}$ & $\cdots$ \\
\hline $5^{\prime}-S S_{-} 1 b$ & $G \cdots \cdots$ & $\cdots \cdots$ & $\cdot-\mathrm{UC}$ & $\cdots \mathrm{C}$ \\
\hline 5'-SS_2 & $\cdots U \cdot$ & $\cdots \cdots$ & & \\
\hline BPS_1 & $\cdots \cdots$ & $\cdots \cdots c$ & $\cdots$ & $\cdot$ \\
\hline 3'-SS_1 & $\cdots \cdots$ & $\cdots \cdots$ & $\cdots \mathrm{C}$ & $\cdots$ \\
\hline 3'-SS_1a & $\cdots \cdots$ & $\cdots$ & $\cdots \mathrm{C}$ & $\cdots \mathrm{C}$ \\
\hline
\end{tabular}

d

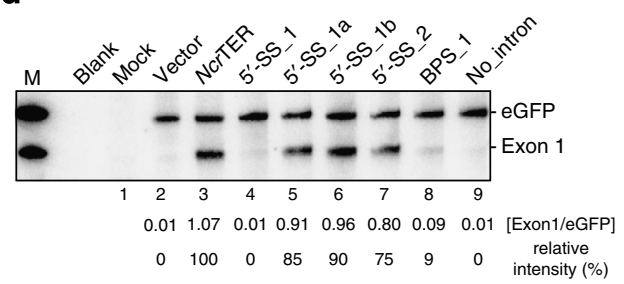

e

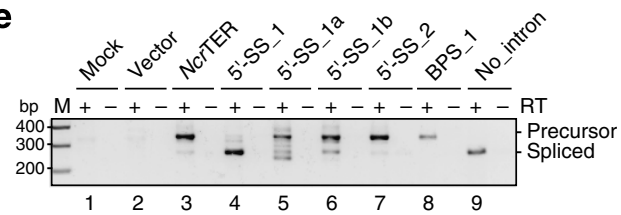

f
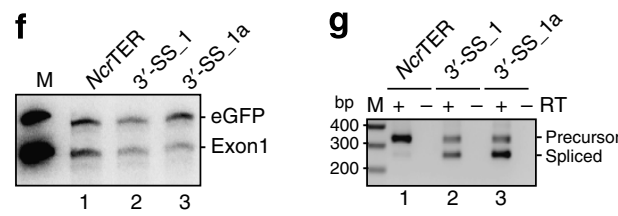

$\begin{array}{lll}1.06 & 0.76 & 0.44\end{array}$ [Exon1/eGFP]

$\begin{array}{llll}100 & 72 & 42 & \text { relative } \\ \text { intensity }(\%)\end{array}$

Figure 3 | Determinants in the NcrTER intron for spliceosomal cleavage. (a) Schematic of U1 and U2 snRNAs base-paired with NcrTER (top, blue) or ccg-1 (bottom, orange) intron 1. (b) Schematic of the dual gene cassette for expressing the NcrTER $3^{\prime}$-end fragment of exon 1, intron, exon 2 and 500 bp

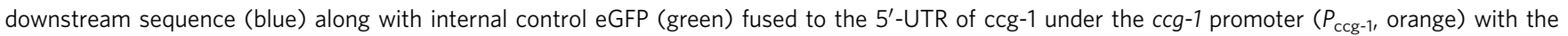
transcription initiation sites shown (black arrows). Annealing positions for riboprobes (Probe) as well as forward (F) and reverse (R) primers for RT-PCR are denoted (purple). (c) Alignment of NcrTER intron mutations (red), mutations to convert the NcrTER intron into the ccg-1 mRNA intron (orange) and a downstream cryptic $3^{\prime}$-SS ( ${ }^{*}$, purple) denoted. The accumulation and splicing variants produced from these intron mutations were evaluated by northern blot analysis $(\mathbf{d}, \mathbf{f})$ and RT-PCR $(\mathbf{e}, \mathbf{g})$. Relative intensity of spliceosomal cleaved ccg-1::NcrTER (Exon 1), determined by the ratio of Exon 1 over eGFP (Exon1/eGFP) with the background of each lane subtracted and relative to the wild-type NcrTER intron, is shown below the blot and the markers (M) were in vitro transcribed ccg-1::NcrTER Exon 1 and ccg-1::eGFP RNA.

As the ccg-1 mRNA intron can be easily converted into the NcrTER-type intron, we examined whether the ccg-1 mRNA intron can be converted for the $S$. pombe-type spliceosomal cleavage by extending the distance between the BPS and $3^{\prime}-\mathrm{SS}$ CAG. Mutant 3'-SS_2 had the proximal 3'-SS eliminated, while mutant Linker_1 introduced a 34-nt insertion between the BPS and the $3^{\prime}$-SS CAG (Fig. 4b). The northern blot and RT-PCR results showed that both mutants had a significantly increased level of mature NcrTER exon-1 RNA (Fig. 4c, lanes 8 and 9) and decreased splicing efficiency (Fig. 4d, lanes 8 and 9). Thus, the ccg-1 mRNA splicing intron can be readily converted to either the NcrTER-type or SpoTER1-type intron for spliceosomal cleavage. We also examined the importance of the BPS in promoting spliceosomal cleavage. Mutant BPS_2 converted the ccg-1 mRNA intron BPS to NcrTER-type intron BPS, while mutant BPS_3 converted it to the strong BPS of spoTER1 intron (Supplementary Figs 3 and $4 \mathrm{~b}$ ). These BPS mutants did not significantly increase the accumulation of mature NcrTER (Fig. 4e) or decrease the splicing efficiency (Fig. 4f) compared with the wild-type ccg-1 mRNA intron.

\section{Discussion}

Telomerase biogenesis requires a $3^{\prime}$-end maturation step for TER, which is remarkably divergent across eukaryotic taxa and utilizes a wide array of distinct mechanisms. Within the phylum Ascomycota, certain budding yeast TERs employ specific transcription termination pathways and nucleolytic cleavage for $3^{\prime}$-end maturation ${ }^{5,30}$, while the fission yeast $S$. pombe and a few select budding yeast species employ spliceosomal cleavage ${ }^{19}$. Herein we have identified a second, yet vastly more widespread, spliceosome-mediated TER $3^{\prime}$-end processing mechanism initially in $N$. crassa and virtually ubiquitous throughout Ascomycete fungi. The N. crassa-type spliceosomal cleavage is mechanistically distinct from the $S$. pombe-type and is dependent on a unique 5'-SS AUAAGU sequence (Fig. 2a and Supplementary Fig. 1). While critical for the S. pombe-type mechanism, the BPS and the distance to the $3^{\prime}$-SS are seemingly unimportant for the N. crassatype. The prevalence of the N. crassa-type TER intron throughout all known Pezizomycotina fungal TERs and within the early diverging Taphrinomycotina fission yeast suggests that the $N$. crassa-type spliceosomal cleavage mechanism is ancestral and the $S$. pombe-type is later evolved.

We propose that the highly prevalent and basal N. crassa-type TER intron is ancestral and the $S$. pombe-type intron is later evolved by an A-to-G substitution of the $5^{\prime}$-SS together with strengthening the BPS, extending distance to the $3^{\prime}-\mathrm{SS}$ and weakening the Py tract. It would seem less likely that multiple TER-intron fusion events occurred during the short evolutionary time within each of the three Ascomycete subphyla. Instead, it is more plausible that two transition events converted the N. crassatype to the $S$. pombe-type intron independently along the fission and budding yeast lineages. The fission yeast transition event seems to have occurred adjacent to the emergence of the basal $S$. japonicas species that employs the ancestral $N$. crassa-type intron (Fig. 5). Similarly, select budding yeast species from the genera Candida and Hansenula harbour the S. pombe-type intron for TER $3^{\prime}$-end maturation (Figs $2 \mathrm{c}$ and 5), while later-branching budding yeast species appear to have lost the spliceosomal cleavage intron entirely. These Saccharomyces species with intron-less TERs instead employ the Nrd1/Nab3-dependent transcription termination pathway for generating non-polyadenylated 
a

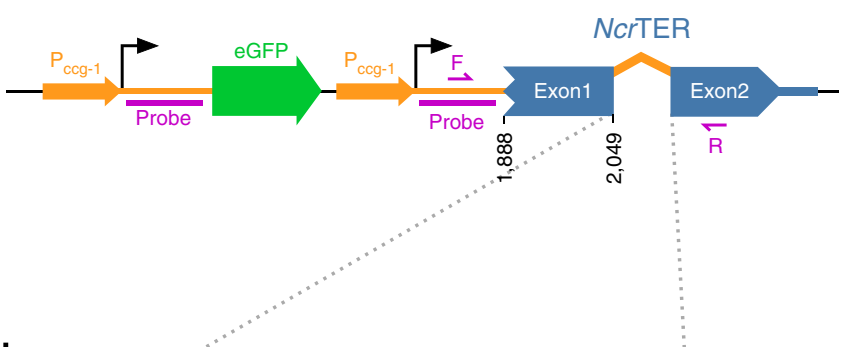

b

\begin{tabular}{|c|c|c|c|c|}
\hline & 5'-SS & BPS & * 3'-SS $^{\prime}$ & * \\
\hline $\operatorname{ccg}-1$ & $\mathrm{G} \cup \mathrm{A} G \mathrm{G} \cup$ & $\mathrm{CAC \cup G} A$ & $\bar{G}_{-2}$ & UA \\
\hline 5'-SS_3 & $A \ldots A$. & $\ldots \ldots$ & & \\
\hline $5^{\prime}$-SS_3a & $A \cdot A \cdot$. & $\ldots \ldots$ & . $\mathrm{G}$ & \\
\hline 5'-SS_3b & $A \cdot A \cdot \cdot$ & $\cdot \cdot \cdot \cdot$ & $\cdot$.U. & . \\
\hline $5^{\prime}-S S \_4$ & A.... & $\cdot \cdot \cdot \cdot$ & & \\
\hline $5^{\prime}-S S \_4 a$ & $A \ldots$ & . . . . & . $G$ & \\
\hline $5^{\prime}-S S \_4 b$ & $A \ldots$ & $\ldots \ldots$ &.$U$. & \\
\hline 3'-SS_2 & $\cdots$ & $\cdots \cdots$ & $\cdots \cup C$ & . \\
\hline Linker_1 & 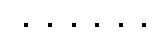 & $\cdots$ & . & \\
\hline BPS_2 & $\ldots$ & UG. . & & \\
\hline BPS_3 & . . . . & U...A. & & \\
\hline
\end{tabular}

C

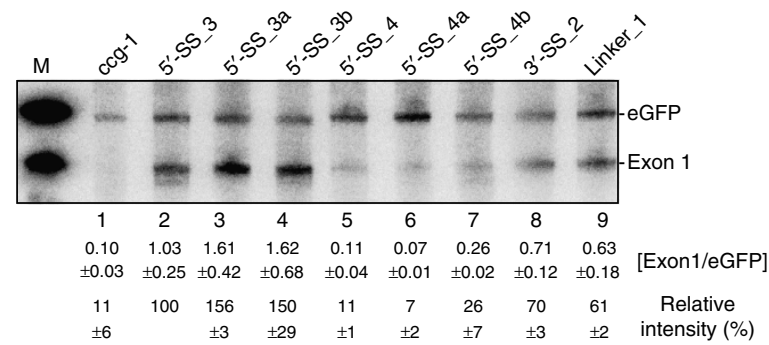

d

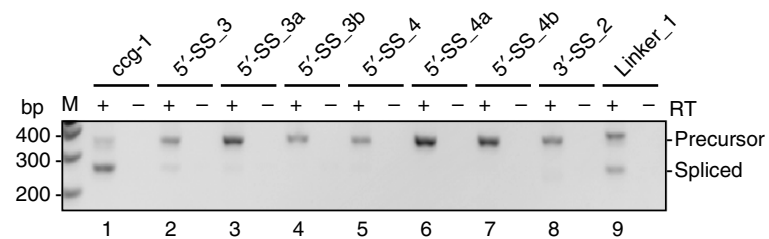

$\mathbf{e}$

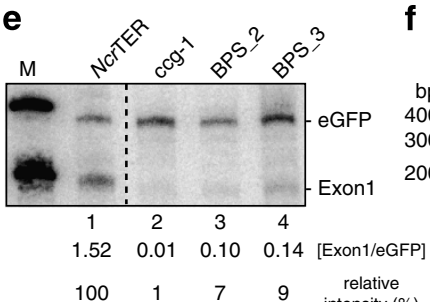

f

ative

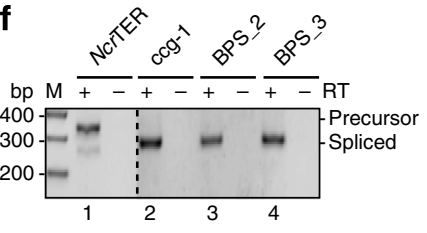

Figure 4 | Conversion of a ccg-1 mRNA intron to the NcrTER-type intron. (a) Schematic of the dual gene cassette for expressing the ccg-1::NcrTER (blue) with the NcrTER intron replaced with the ccg-1 mRNA intron (orange line). (b) Alignment of ccg-1 mRNA intron mutations (red), mutations to convert ccg-1 mRNA intron into the NcrTER-like intron (blue) and two cryptic $3^{\prime}-\mathrm{SS}\left({ }^{*}\right.$, purple) denoted. The accumulation and splicing variants produced from these intron mutations were evaluated by northern blot analysis $(\mathbf{c}, \mathbf{e})$ and RT-PCR $(\mathbf{d}, \mathbf{f})$. Average relative intensity of two independent replicates with s.e. (c) and relative intensity (e) of spliceosomal cleaved ccg-1::NcrTER (Exon 1), determined by the ratio of Exon 1 over eGFP (Exon1/eGFP) with the background of each lane subtracted and relative to the mutant $5^{\prime}$-SS_3 or wild-type NcrTER intron, is shown below the blot and the markers (M) were in vitro transcribed ccg-1::NcrTER Exon 1 and ccg-1::eGFP RNA.

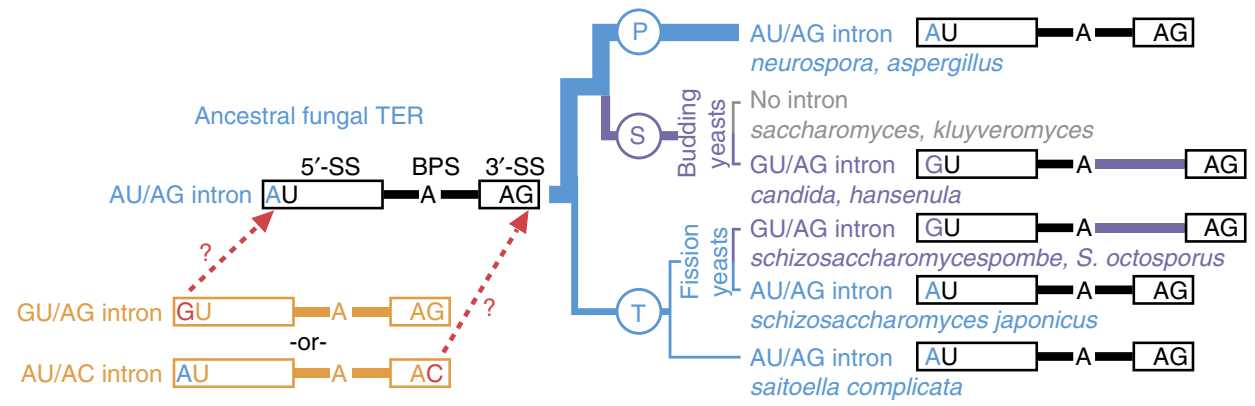

Figure 5 | An evolutionary history of the fungal TER intron. The AU/AG intron is predominate and basal (thick blue line) among fungal TER introns. The GU/AG or AU/AC (U1/U2-type) introns (orange) are the putative predecessor of the fungal TER AU/AG intron with only a single point mutation (red) in either intron necessary to convert into this intron type (dashed red lines). From this putative ancestral fungal TER AU/AG intron, the TER GU/AG type intron appears to have arisen twice independently (purple). A subgroup of budding yeasts has lost TER spliceosomal cleavage.

TERs $^{30}$. Two independent transition events from a more ancestral $N$. crassa-type TER intron to the $S$. pombe-type or the loss of TER intron explain the scattered presence of the $S$. pombe-type intron within Ascomycete fungal TERs and the uniform presence of the $N$. crassa-type intron.

While there is strong support for the ancestry of the N. crassatype TER intron, there are two conceivable possibilities for the origin of the progenitor fungal TER intron. The progenitor fungal TER intron probably arose from a single gene fusion event between an intron-less fungal TER gene and either a common GU/AG or a rare AU/AC mRNA intron (Fig. 5). This newly intron-containing TER gene then evolved into the N. crassa-type and later twice into the $S$. pombe-type TER intron. Deriving the $N$. crassa-type TER intron from a GU/AG intron requires alterations in the $5^{\prime}$-SS, while adoption from an AU/AC intron necessitates a C-to-G substitution of the last residue in the $3^{\prime}$-SS. Our mutagenesis studies demonstrate that the NcrTER spliceosomal cleaving intron can be readily converted to either a canonical GU/AG or a rare AU/AC intron-permitting efficient splicing-with only single or double mutations necessary (Figs 3 and 4). Previous studies in yeast have shown that an introduced G-to-A substitution of the first residue in the $5^{\prime}$-SS of a common GU/AG mRNA intron strongly inhibits the second step in splicing $^{25,31}$. Arising from an AU/AC intron would negate a G-to-A transition substitution and instead require a $3^{\prime}$-SS mutation for efficient spliceosomal cleavage. Given the abundance of GU/AG mRNA intron in fungal genomes ${ }^{32}$, we favour the possibility that the N. crassa-type intron evolved from 
a GU/AG mRNA intron. While all three NcrTER variants reconstituted equal telomerase activity in vitro (Supplementary Fig. 5), it is evident that a spliceosomal cleaving TER intron is evolutionarily selected for as it removes the poly(A) tail from TER, which presumably prevents undesired cytoplasmic localization or poly(A) tail-mediated degradation ${ }^{33}$. Therefore, spliceosomal cleavage may promote nuclear retention and accumulation of functional TER. In $S$. pombe, disruption of TER spliceosome-mediated $3^{\prime}$-end processing results in reduced accumulation of SpoTER1 and telomere shortening ${ }^{18}$. The identification of additional fungal TERs from more basal species is necessary to determine the evolutionary origins of fungal TER introns.

The identification of the widespread N. crassa-type spliceosomal cleavage mechanism within fungal TERs provides insights into the evolution of TER $3^{\prime}$-end processing. Spliceosomal cleavage was initially viewed as a molecular idiosyncrasy, unique to a single RNA from a single species. Our findings demonstrate that spliceosomal cleavage is the dominant mechanism for Ascomycete fungal TER $3^{\prime}$-end processing, found in the vast majority of species with known TERs. It has yet to be determined whether this mechanism is conserved outside of the Ascomycota phylum, due to the complete lack of TERs identified from the Basidiomycota phylum. Moreover, it is unlikely that this RNA processing mechanism is exclusive to TER and is potentially employed by other non-coding RNAs for $3^{\prime}$-end maturation. This work expands our understanding of the diversity of spliceosomal cleavage mechanisms, the origins and evolution of TER maturation, as well as our knowledge of non-coding RNA processing and biogenesis.

\section{Methods}

Fungal strains. $N$. crassa (FGSC 2489 wild type and FGSC $9720 \mathrm{NC1}$ his-3 mutant) and $A$. nidulans (FGSC A4 wild type) were obtained from the Fungal Genetics Stock Center (FGSC). N. crassa cells were grown at $30^{\circ} \mathrm{C}$ with continuous light exposure in Vogel's liquid minimal media supplemented with $2 \%$ sucrose $^{34}$. A. nidulans cells were grown at $37^{\circ} \mathrm{C}$ in YG liquid medium $(0.5 \%$ yeast extract, $2 \%$ dextrose, $0.1 \% \mathrm{v} / \mathrm{v}$ Cove's trace elements and $0.12 \% \mathrm{MgSO}_{4} \bullet 7 \mathrm{H}_{2} \mathrm{O}$ ). M. graminicola cells (strain IPO323) were obtained from Dr Gert Kema and grown at $18^{\circ} \mathrm{C}$ in yeast glucose broth (1\% yeast extract and $3 \%$ glucose). S. complicata (strain Y-17804), S. japonicus (strain Y-1361) and S. octosporus (strain Y-855) were obtained from ARS Culture Collection (NRRL) and grown at room temperature in YM broth $(0.3 \%$ yeast extract, $0.3 \%$ malt extract, $0.5 \%$ peptone and $1 \%$ glucose).

\section{Identification of Schizosaccharomyces TER sequences. Two protein-coding} genes (sub1, SPAC16A10.03c and rho4, dad1) flanking the SpoTER1 gene served as queries searching against S. japonicus (strain yFS275) and S. octosporus (strain yFS286) genomic sequencing data downloaded from the Broad Institute Database $^{35}$. The intervening sequences between these putative protein coding genes were searched using the program Infernal ${ }^{36}$ with a covariance model derived from the sequence alignment of Ascomycete TER three-way junctions ${ }^{22}$ and yielded a unique hit for each species. The $5^{\prime}$ - and $3^{\prime}$-ends of TERs from $S$. japonicus and S. octosporus were determined by RACE using the FirstChoice RLM-RACE kit (Life technologies). These cloned TER cDNAs sequences were deposited to GenBank with accession numbers KJ740640 (S. japonicas) and KJ740641 (S. octosporus)

Generation of $\boldsymbol{N}$. crassa recombinant strains. The expression cassette containing the $e G F P$ gene and the NcrTER 3 '-portion that includes exon-1 (residues $1,888-2,049$ ), the 74-residue intron (residues 2,050-2,123), exon 2 (residues 2,124-2,200) and the $500 \mathrm{bp}$ downstream flanking genomic sequence that contains a polyadenylation signal was constructed by overlapping PCR and cloned into the pCCG::NcrTER vector ${ }^{22,37}$. The $e G F P$ gene and the NcrTER $3^{\prime}$-portion were each fused immediately downstream of the ccg-1 $5^{\prime}$-UTR and expressed under separate ccg-1 promoters. Intron mutants were generated by site-directed mutagenesis of this parental construct. For $N$. crassa transformation, the preserved conidia of N. crassa strain FGSC $9720 \mathrm{NC1}$ (mat A his-3 4 mus-52::bar ${ }^{+}$) were inoculated into $25 \mathrm{ml}$ Vogel's minimal solid media in a $500-\mathrm{ml} \mathrm{flask}$. After growing at $30^{\circ} \mathrm{C}$ for 3 days in total darkness, the mycelia were exposed to continuous light for 7 days. Conidia were harvested with $30 \mathrm{ml} 1 \mathrm{M}$ sorbitol and filtered through two layers of cheese cloth. Conidia were collected by centrifugation at $3,000 \mathrm{~g}$ for $3 \mathrm{~min}$ at $4{ }^{\circ} \mathrm{C}$ and washed three times with $1 \mathrm{M}$ sorbitol. After the final wash the conidia were suspended in $1 \mathrm{M}$ sorbitol to $\mathrm{OD}_{600}$ of $0.5-1.0$. Conidia were transformed with $1 \mu \mathrm{g}$ of Stu I-linearized wild-type or mutant plasmid DNA by electroporation using a Gene Pulser Xcell system (Bio-Rad) set to 1,500 volts and $25 \mu$ F. Selection of his $^{+}$transformants was performed in Vogel's minimum medium supplemented with $2 \%$ sorbose in place of sucrose. Following growth at $30^{\circ} \mathrm{C}$ for 3 days, the colonies were inoculated onto Vogel's slants. Screening of homokaryon strains was performed by Southern blot analysis.

Southern blot analysis. Genomic DNA was isolated from $20 \mathrm{mg}$ N. crassa mycelia using the Wizard Genomic DNA purification kit (Promega). The mycelia were lysed in $700 \mu \mathrm{l}$ nuclei lysis solution in a $1.5 \mathrm{ml}$ screw top tube containing $300 \mathrm{mg}$ of 0.5 - mm zirconia/silica beads (BioSpec Products) using a MiniBeadBeater-16 (BioSpec Products) at 3,450 r.p.m. for $1 \mathrm{~min}$. The homogenates were incubated at $65^{\circ} \mathrm{C}$ for $15 \mathrm{~min}$ and treated with $1 \mu \mathrm{l} \mathrm{RNase} \mathrm{A}\left(50 \mathrm{mg} \mathrm{ml}{ }^{-1}\right)$ at $37^{\circ} \mathrm{C}$ for $30 \mathrm{~min}$. Following RNase treatment, $220 \mu \mathrm{l}$ of protein precipitation buffer (Promega) was added, followed by vortexing vigorously for $20 \mathrm{~s}$ and incubation on ice for $5 \mathrm{~min}$. After centrifugation at $16,000 \mathrm{~g}$ for $5 \mathrm{~min}$, genomic DNA was precipitated by isopropanol and pelleted by centrifugation at $16,000 \mathrm{~g}$ for $1 \mathrm{~min}$. The DNA pellet was washed once with $75 \%$ ethanol, air dried and suspended in $100 \mu \mathrm{TE}$ buffer (10 mM Tris- $\mathrm{HCl} \mathrm{pH} 8.0$ and $1 \mathrm{mM}$ EDTA pH 8.0). Ten micrograms of genomic DNAs were digested with $10 \mathrm{U}$ BspEI (New England Biolabs) in a $100 \mu \mathrm{l}$ reaction at $37^{\circ} \mathrm{C}$ overnight. Digested genomic DNA was phenol/chloroform extracted, ethanol precipitated and resolved on a $0.8 \%$ agarose gel. The agarose gel was denatured by immersion in a solution of $1.5 \mathrm{M} \mathrm{NaCl}$ and $0.5 \mathrm{M} \mathrm{NaOH}$ for $20 \mathrm{~min}$ twice, followed by neutralization in a solution of $1.5 \mathrm{M} \mathrm{NaCl}$ and $0.5 \mathrm{M}$ Tris- $\mathrm{HCl} \mathrm{pH} 7.5$ for $15 \mathrm{~min}$ twice. The gel was dried in a gel dryer and pre-hybridized in $20 \mathrm{ml}$ hybridization solution $\left(5 \times \mathrm{SSC}, 5 \times\right.$ Denhardt's reagent, $0.1 \%$ SDS and $20 \mathrm{mM} \mathrm{NaH}_{2} \mathrm{PO}_{4}, \mathrm{pH}$ $7.0)$ at $58^{\circ} \mathrm{C}$ for $1 \mathrm{~h}$. In-gel hybridization was performed at $58^{\circ} \mathrm{C}$ overnight in $15 \mathrm{ml}$ fresh hybridization solution with a ${ }^{32} \mathrm{P}$ end-labelled oligonucleotide probe targeting the his-3 locus. The gel was then washed at $58^{\circ} \mathrm{C}$ for 20 min three times with a solution of $3 \times$ SSC and $0.1 \%$ SDS and three times with a solution of $2 \times$ SSC and $0.1 \%$ SDS. The gel was exposed to a phosphorstorage screen and imaged on a FX-Pro phosphorimager (Bio-Rad)

Northern blot analysis. Total RNA was isolated from $N$. crassa mycelia grown in Vogel's minimum medium at $30^{\circ} \mathrm{C}$ with continuous light exposure for expressing ccg1::eGFP and ccg1::NcrTER fusion genes under the light-inducible $c c g-1$ promoter. Twenty milligrams of mycelia were homogenized in $1 \mathrm{ml}$ TRI-reagent (Molecular Research Center) at room temperature using $300 \mathrm{mg} 0.5-\mathrm{mm}$ zirconia/ silica beads (BioSpec Products) and a MiniBeadBeater-16 (BioSpec Products) at 3,450 r.p.m. for $1 \mathrm{~min}$. Homogenate was supplemented with $0.1 \mathrm{ml} 1$-bromo-3chloropropane, vigorously mixed and centrifuged at $4{ }^{\circ} \mathrm{C}$ for phase separation. Residual phenol in the aqueous phase was removed by chloroform extraction followed by ethanol precipitation. For RNase H cleavage of ccg1::eGFP mRNA to remove the heterogeneous poly(A) tail, $16 \mu \mathrm{g}$ total RNA was annealed with 600 pmol of DNA oligo (5'-AAGTCGTGCTGCTTCATGTG-3') by incubation at $65^{\circ} \mathrm{C}$ for $5 \mathrm{~min}$ and slowly cooled to room temperature. The RNA/DNA hybrid was digested with $5 \mathrm{U}$ of RNase $\mathrm{H}$ (New England Biolabs) at $37^{\circ} \mathrm{C}$ for $30 \mathrm{~min}$ followed by ethanol-precipitation. RNase $\mathrm{H}$-treated total RNA was resolved on a $4 \%$ polyacrylamide/ $8 \mathrm{M}$ urea gel, electrotransferred and UV crosslinked to a Hybond-XL membrane (GE Healthcare). For the full-length TER, untreated total RNA was resolved on a $1.5 \%$ agarose/formaldehyde denaturing gel, capillary transferred overnight and UV crosslinked to a Hybond-XL membrane (GE Healthcare). The membrane was pre-hybridized in ULTRAhyb hybridization buffer (Life technologies) at $65^{\circ} \mathrm{C}$ for $30 \mathrm{~min}$ and hybridized overnight with a ${ }^{32} \mathrm{P}$ radio-labelled riboprobe targeting the ccg-1 $5^{\prime}$-UTR fused to the $e G F P$ mRNA and the NcrTER $3^{\prime}$-fragment. The blot was washed at $65^{\circ} \mathrm{C}$ twice in a solution of $1 \times$ SSC and $0.2 \%$ SDS for $10 \mathrm{~min}$, and twice in a solution of $0.2 \times$ SSC and $0.1 \%$ SDS for $30 \mathrm{~min}$. The blot was exposed to a phosphorstorage screen and imaged on a FX-Pro phosphorimager (Bio-Rad).

RT-PCR. Total RNA was treated with RNase-free DNase I (Zymo Research Corporation) to remove any genomic DNA contamination, followed by phenol/ chloroform extraction and ethanol precipitation. For cDNA synthesis, $2 \mu \mathrm{g}$ DNase I-treated total RNA was annealed with 100 pmol oligo $d(T)_{25}$ DNA primer in the presence of $10 \mathrm{pmol}$ each dNTP by incubation at $65^{\circ} \mathrm{C}$ for $3 \mathrm{~min}$ and quickly cooled on ice. A $20 \mu \mathrm{l}$ reverse-transcription reaction was performed in $1 \times$ Protoscript II Reverse Transcriptase Reaction buffer $(50 \mathrm{mM}$ Tris- $\mathrm{HCl}$ pH 8.3, $75 \mathrm{mM}$ $\mathrm{KCl}$ and $3 \mathrm{mM} \mathrm{MgCl}_{2}$ ), $10 \mathrm{mM}$ DTT and $200 \mathrm{U}$ ProtoScript II Reverse Transcriptase (New England Biolabs) at $48^{\circ} \mathrm{C}$ for $30 \mathrm{~min}$. The reaction was terminated by incubation at $80^{\circ} \mathrm{C}$ for $5 \mathrm{~min}$. PCR was performed in a $25-\mu \mathrm{l}$ reaction with $1 \mu \mathrm{l}$ of $1 / 2 \times$ diluted reverse-transcription reaction in $1 \times$ Q5 Reaction buffer $(25 \mathrm{mM}$ TAPS- $\mathrm{HCl} \mathrm{pH} \mathrm{9.3,50} \mathrm{mM} \mathrm{KCl}, 2 \mathrm{mM} \mathrm{MgCl}_{2}$ and $1 \mathrm{mM} \beta$-mercaptoethanol), $0.2 \mathrm{mM}$ each dNTP, $0.5 \mathrm{U}$ of Q5 DNA Polymerase, $0.2 \mu \mathrm{M}$ forward primer $\left(5^{\prime}\right.$-CACAACACTCAAACCACTTTCAC- $\left.3^{\prime}\right)$ and $0.2 \mu \mathrm{M}$ reverse (5'-TGTTCGA CACCGGTCACACT- $3^{\prime}$ ) primer. PCR products were resolved on a $3 \%$ agarose gel. The DNA bands corresponding to the spliced products were gel extracted using a Wizard SV gel and PCR clean-up system (Promega) and sequenced. 


\section{References}

1. Zakian, V. A. The ends have arrived. Cell 139, 1038-1040 (2009).

2. Podlevsky, J. D. \& Chen, J. J.-L. It all comes together at the ends: telomerase structure, function, and biogenesis. Mutat. Res. 730, 3-11 (2012).

3. Chen, J.-L. \& Greider, C. W. Telomerase RNA structure and function: implications for dyskeratosis congenita. Trends Biochem. Sci. 29, 183-192 (2004).

4. Podlevsky, J. D., Bley, C. J., Omana, R. V., Qi, X. \& Chen, J. J.-L. The telomerase database. Nucleic Acids Res. 36, D339-D343 (2008).

5. Chapon, C., Cech, T. R. \& Zaug, A. J. Polyadenylation of telomerase RNA in budding yeast. RNA 3, 1337-1351 (1997).

6. Romero, D. P. \& Blackburn, E. H. A conserved secondary structure for telomerase RNA. Cell 67, 343-353 (1991).

7. Blasco, M. A., Funk, W., Villeponteau, B. \& Greider, C. W. Functional characterization and developmental regulation of mouse telomerase RNA. Science 269, 1267-1270 (1995).

8. Feng, J. et al. The RNA component of human telomerase. Science 269, 1236-1241 (1995)

9. Venteicher, A. S. et al. A human telomerase holoenzyme protein required for Cajal body localization and telomere synthesis. Science 323, 644-648 (2009).

10. Egan, E. D. \& Collins, K. An enhanced H/ACA RNP assembly mechanism for human telomerase RNA. Mol. Cell Biol. 32, 2428-2439 (2012).

11. Hughes, T. R., Evans, S. K., Weilbaecher, R. G. \& Lundblad, V. The Est3 protein is a subunit of yeast telomerase. Curr. Biol. 10, 809-812 (2000).

12. Tang, W., Kannan, R., Blanchette, M. \& Baumann, P. Telomerase RNA biogenesis involves sequential binding by Sm and Lsm complexes. Nature 484, 260-264 (2012)

13. Seto, A. G., Zaug, A. J., Sobel, S. G., Wolin, S. L. \& Cech, T. R. Saccharomyces cerevisiae telomerase is an Sm small nuclear ribonucleoprotein particle. Nature 401, 177-180 (1999).

14. Kannan, K., Nelson, A. D. L. \& Shippen, D. E. Dyskerin is a component of the Arabidopsis telomerase RNP required for telomere maintenance. Mol. Cell Biol. 28, 2332-2341 (2008)

15. Cifuentes-Rojas, C., Kannan, K., Tseng, L. \& Shippen, D. E. Two RNA subunits and POT1a are components of Arabidopsis telomerase. Proc. Natl Acad. Sci. USA 108, 73-78 (2011).

16. Singh, M. et al. Structural basis for telomerase RNA recognition and RNP assembly by the holoenzyme La family protein p65. Mol. Cell 47, 16-26 (2012).

17. Egan, E. D. \& Collins, K. Biogenesis of telomerase ribonucleoproteins. RNA 18, 1747-1759 (2012).

18. Box, J. A., Bunch, J. T., Tang, W. \& Baumann, P. Spliceosomal cleavage generates the 3' end of telomerase RNA. Nature 456, 910-914 (2008).

19. Kannan, R. et al. Intronic sequence elements impede exon ligation and trigger a discard pathway that yields functional telomerase RNA in fission yeast. Genes Dev. 27, 627-638 (2013)

20. Leonardi, J., Box, J. A., Bunch, J. T. \& Baumann, P. TER1, the RNA subunit of fission yeast telomerase. Nat. Struct. Mol. Biol. 15, 26-33 (2008).

21. Horowitz, D. S. The mechanism of the second step of pre-mRNA splicing Wiley Interdiscip. Rev. RNA 3, 331-350 (2012).

22. Qi, X. et al. The common ancestral core of vertebrate and fungal telomerase RNAs. Nucleic Acids Res. 41, 450-462 (2013).

23. Gunisova, S. et al. Identification and comparative analysis of telomerase RNAs from Candida species reveal conservation of functional elements. RNA 15, 546-559 (2009).

24. Smekalova, E. M. et al. Specific features of telomerase RNA from Hansenula polymorpha. RNA 19, 1563-1574 (2013).
25. Newman, A. J., Lin, R. J., Cheng, S. C. \& Abelson, J. Molecular consequences of specific intron mutations on yeast mRNA splicing in vivo and in vitro. Cell $\mathbf{4 2}$, 335-344 (1985)

26. Freitag, M., Hickey, P. C., Raju, N. B., Selker, E. U. \& Read, N. D. GFP as a too to analyze the organization, dynamics and function of nuclei and microtubules in Neurospora crassa. Fungal Genet. Biol. 41, 897-910 (2004).

27. Hall, S. L. \& Padgett, R. A. Requirement of U12 snRNA for in vivo splicing of a minor class of eukaryotic nuclear pre-mRNA introns. Science 271, 1716-1718 (1996).

28. Tarn, W. Y. \& Steitz, J. A. A novel spliceosome containing U11, U12, and U5 snRNPs excises a minor class (AT-AC) intron in vitro. Cell 84, 801-811 (1996).

29. Tarn, W. Y. \& Steitz, J. A. Highly diverged U4 and U6 small nuclear RNAs required for splicing rare AT-AC introns. Science 273, 1824-1832 (1996).

30. Noel, J. F., Larose, S., Abou Elela, S. \& Wellinger, R. J. Budding yeast telomerase RNA transcription termination is dictated by the Nrd1/Nab3 non-coding RNA termination pathway. Nucleic Acids Res. 40, 5625-5636 (2012).

31. Nelson, K. K. \& Green, M. R. Mechanism for cryptic splice site activation during pre-mRNA splicing. Proc. Natl Acad. Sci. USA 87, 6253-6257 (1990).

32. Kupfer, D. M. et al. Introns and splicing elements of five diverse fungi. Eukaryot. Cell 3, 1088-1100 (2004).

33. Callahan, K. P. \& Butler, J. S. TRAMP complex enhances RNA degradation by the nuclear exosome component Rrp6. J. Biol. Chem. 285, 3540-3547 (2010).

34. Vogel, H. J. A convenient growth medium for Neurospora (Medium N). Microbial Genet. Bull. 13, 42-43 (1956)

35. Rhind, N. et al. Comparative functional genomics of the fission yeasts. Science 332, 930-936 (2011)

36. Nawrocki, E. P., Kolbe, D. L. \& Eddy, S. R. Infernal 1.0: inference of RNA alignments. Bioinformatics 25, 1335-1337 (2009).

37. Honda, S. \& Selker, E. U. Tools for fungal proteomics: multifunctional neurospora vectors for gene replacement, protein expression and protein purification. Genetics 182, 11-23 (2009).

\section{Acknowledgements}

We thank Dr Soo-Chen Cheng for critical reading of the manuscript. This work was supported by National Institutes of Health Grant GM094450 to J.J.-L.C.

\section{Author contributions}

X.Q. and J.J.-L.C. designed the experiments; X.Q., D.P.R., J.D.P. and Y.L. carried out the experiments, A.M. and P.F.S. performed bioinformatics analysis. X.Q., J.D.P. and J.J.-L.C. analysed the data and wrote the paper.

\section{Additional information}

Accession codes: The sequences of S. japonicas and S. octosporus TERs were deposited to GenBank with accession numbers KJ740640 and KJ740641, respectively.

Supplementary Information accompanies this paper at http://www.nature.com/ naturecommunications

Competing financial interests: The authors declare no competing financial interests.

Reprints and permission information is available online at http://npg.nature.com/ reprintsandpermissions/

How to cite this article: Qi, X. et al. Prevalent and distinct spliceosomal $3^{\prime}$-end processing mechanisms for fungal telomerase RNA. Nat. Commun. 6:6105 doi: 10.1038/ncomms7105 (2015). 